\title{
An Evidence Review of Low-Value Care Recommendations: Inconsistency and Lack of Economic Evidence Considered
}

\author{
David D. Kim, $P h D^{1,2}{ }^{\infty}$, Lauren A. Do, BS ${ }^{7}$, Allan T. Daly, $M S^{7}$, John B. Wong, MD², \\ James D. Chambers, $P h D^{1,2}$, Daniel A. Ollendorf, $P h D^{1,2}$, and Peter J. Neumann, $S c D^{1,2}$
}

'Center for the Evaluation of Value and Risk in Health, Institute for Clinical Research and Health Policy Studies, Tufts Medical Center, 800 Washington St., Box 063, Boston, MA, USA; ${ }^{2}$ Department of Medicine, Tufts University School of Medicine, Boston, MA, USA; ${ }^{3}$ Division of Clinical Decision Making, Tufts Medical Center, Boston, MA, USA.

BACKGROUND: Low-value care, typically defined as health services that provide little or no benefit, has potential to cause harm, incur unnecessary costs, and waste limited resources. Although evidence-based guidelines identifying low-value care have increased, the guidelines differ in the type of evidence they cite to support recommendations against its routine use.

OBJECTIVE: We examined the evidentiary rationale underlying recommendations against low-value interventions.

DESIGN: We identified 1167 "low-value care" recommendations across five US organizations: the US Preventive Services Task Force (USPSTF), the "Choosing Wisely" Initiative, American College of Physicians (ACP), American College of Cardiology/American Heart Association (ACC/ AHA), and American Society of Clinical Oncology (ASCO). For each recommendation, we classified the reported evidentiary rationale into five groups: (1) low economic value; (2) no net clinical benefit; (3) little or no absolute clinical benefit; (4) insufficient evidence; (5) no reason mentioned. We further investigated whether any cited or otherwise available cost-effectiveness evidence was consistent with conventional low economic value benchmarks (e.g., exceeding $\$ 100,000$ per quality-adjusted life-year).

RESULTS: Of the identified low-value care recommendations, Choosing Wisely contributed the most $(N=582$, $50 \%)$, followed by ACC/AHA ( $N=250,21 \%)$. The services deemed "low value" differed substantially across organizations. "No net clinical benefit" ( $N=428,37 \%)$ and "little or no clinical benefit" ( $N=296,25 \%)$ were the most commonly reported reasons for classifying an intervention as low value. Consideration of economic value was less frequently reported $(N=171,15 \%)$. When relevant costeffectiveness studies were available, their results were mostly consistent with low-value care recommendations. CONCLUSIONS: Our study found that evidentiary rationales for low-value care vary substantially, with most

This paper was previously presented at the Virtual 2020 Academy Health Annual Research Meeting.

Supplementary Information The online version contains supplementary material available at https://doi.org/10.1007/s11606-021-06639-2.

Received September 22, 2020

Revised December 11, 2020

Accepted January 25, 2021

Published online February 23, 2021 recommendations relying on clinical evidence. Broadening the evidence base to incorporate cost-effectiveness evidence can help refine the definition of "low-value" care to reflect whether an intervention's costs are worth the benefits. Developing a consensus grading structure on the strength and evidentiary rationale may help improve de-implementation efforts for low-value care.

KEYWORDS: low-value care; guidelines; cost-effectiveness; evidence.

J Gen Intern Med 36(1 1):3448-55

DOI: $10.1007 /$ s11606-021-06639-2

(C) Society of General Internal Medicine 2021

\section{INTRODUCTION}

An estimated $\$ 100$ billion is spent on low-value health care in the USA. ${ }^{1}$ Low-value care, typically defined as health services that provide little or no benefit, has potential to cause harm, incur unnecessary costs, or waste limited healthcare resources." 2,3 Various organizations, including the "Choosing Wisely" Initiative from the American Board of Internal Medicine Foundation, ${ }^{4}$ have developed evidence-based guidelines identifying low-value care, and the number and scope of these guidelines have increased. ${ }^{5}$ Although modest declines have been observed in selected services, ${ }^{6-8}$ the provision of lowvalue care remains prevalent with substantial variations by service types, physician characteristics, provider organizations, and geographic regions. ${ }^{9-13}$

Experts have emphasized that a deliberate, multi-pronged approach that involves changing financial incentives and delivering better information for both providers and patients is warranted to reduce low-value care. ${ }^{14-16}$ The process should necessarily start with identifying evidence of low-value care and understanding the rationale for calling a service "low value." However, despite the importance of evidence-based guidelines, the contribution of clinical versus economic evidence to justify characterization as low-value care is often unclear. ${ }^{17-19}$ We reviewed "low-value care" guideline recommendations across five US organizations to examine the role played by clinical and economic evidence to justify the inclusion of low-value services. 


\section{METHODS}

\section{Data Sources}

We reviewed guidelines across five major organizations: the US Preventive Services Task Force (USPSTF), the "Choosing Wisely" Initiative (CW), American College of Physicians (ACP), American College of Cardiology/American Heart Association (ACC/AHA), and American Society of Clinical Oncology (ASCO). The guidelines were current as of February 26, 2020. We selected these organizations because they reflect major US initiatives to reduce low-value care, with their guidelines employing evidence grading systems (USPSTF, ACC/AHA) or including evidence-based recommendation statements (CW, ACP, ASCO).

After identifying 1361 "low-value care" recommendations across five organizations, we excluded 194 that did not refer to a specific procedure (e.g., general recommendations, such as "Do not prescribe an expensive, brand name drug when less costly, equally effective options are available"). For each recommendation, we extracted information pertaining to disease area (using the International Classification of Diseases, Tenth Revision, Clinical Modification [ICD-10-CM]), intervention type, target population, source of the recommendation, publication date, and cited references. We then examined the distribution of low-value care recommendations by the source, disease area, and intervention type. We also investigated the extent to which guidelines for low-value care were consistent across organizations and their recommendations.

\section{Evidence Review}

For the final sample of 1167 low-value care recommendations, we classified the reported evidentiary rationale into five mutually exclusive hierarchical groups: (1) low economic value (e.g., unfavorable cost-effectiveness, high price, or related economic terms, including costs and efficiency); (2) no net clinical benefit (i.e., the risk of harms outweigh the benefits); (3) little or no absolute clinical benefit; (4) insufficient evidence; (5) no reason mentioned (Fig. 1). These classes were assigned hierarchically by their inclusiveness of additional evidence rather than by the superiority or importance of the recommendations. For example, Class I (the most inclusive category) considers additional economic evidence alongside clinical evidence.

The type of economic evidence included empirical studies, systematic reviews, and meta-analyses of cost analyses, costeffectiveness analyses, and budget impact analyses. Among identified economic evidence, we determined whether the cited evidence was relevant to the specific recommendation based on the intervention and comparator(s), disease classification, population, and settings (e.g., hospital, nursing home).

Two researchers (LAD, ATD) independently reviewed each recommendation and classified the evidentiary rationale reported in the recommendation statement. (See the decision chart used in our assessment of the evidentiary rationale in Online Supplement Figure 1.) We calculated Kappa statistics to measure the degree of agreement among reviewers. ${ }^{20}$ After the initial independent assessment, the two reviewers' level of agreement was moderate (Kappa statistic $=0.609$ and percentage agreement $=70 \%$ ). However, the level of the agreement varied across guidelines, ranging from almost perfect for the USPSTF guidelines (Kappa statistic $=0.953$ ) to minimal for the ACP guidelines (Kappa statistic $=0.314)$ (Online Supplement Table 1). Consensus meetings with a third researcher (DDK) resolved any initial disagreements.

\section{Cost-effectiveness Evidence Used in Low- Value Care Recommendations}

As a secondary analysis, we examined the consistency between available cost-effectiveness evidence and low-value recommendations. Cost-effectiveness analysis (CEA) is an analytic tool that explicitly quantifies the comparative benefits and costs among alternative interventions. ${ }^{21,22}$ It helps to illuminate whether the additional health produced by an intervention (over an alternative) justifies its additional cost. ${ }^{23}$

For each of the low-value care recommendations, we reviewed the accompanying list of references to identify cost-effectiveness evidence cited to support the claims, regardless of whether "low economic value" was explicitly mentioned as the evidentiary rationale. We then further investigated whether any cited or other available cost-effectiveness evidence for low-value care recommendations was consistent with cost-effectiveness criteria. In addition to each CEA cited in the guideline's recommendations, we searched the Tufts Medical Center CEA Registry (a database of over 8000 published cost-per-quality-adjusted-life-year [QALY] studies from 1974 through 2019; available at www.cearegistry.org) for relevant cost-effectiveness evidence. ${ }^{24}$

When the identified CEAs were deemed relevant to the details of the recommendations (e.g., disease areas, intervention and comparator(s), target population), we extracted the incremental cost-effectiveness ratios (ICER) and cost-effectiveness threshold (e.g., \$100,000 per QALY) cited in the study. Based on the study author's judgment on cost-effectiveness, we evaluated whether the cost-effectiveness evidence was consistent with the "lowvalue" care recommendation, according to the following consistency criteria: (1) when the intervention was less effective and more costly than its comparator (i.e., "dominated"); (2) when the intervention was deemed "not cost-effective"; or (3) when more cost-effective alternatives were available. For example, when an intervention's ICER exceeds a $\$ 100,000$ per QALY threshold, we concluded that the cost-effectiveness evidence is consistent with the guideline recommendations, regardless of whether the cost-effectiveness evidence is explicitly mentioned in the recommendation statements.

\section{RESULTS}

\section{Analysis of Low-Value Care Recommendations}

Of the 1167 identified low-value care recommendations, Choosing Wisely contributed the most $(N=582,50 \%)$, 


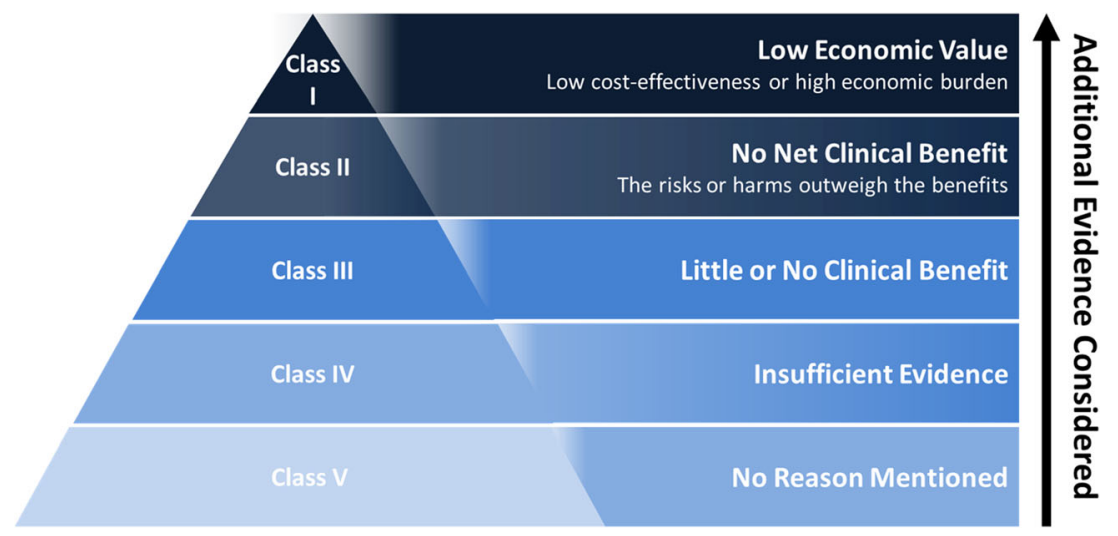

Figure 1 Classification of evidentiary rationale for low-value care. Note: In the pyramid scheme of our classification system, the classes are not hierarchical by superiority or importance, but by inclusivity. For example, Class I recommendations consider additional economic evidence alongside clinical evidence (net clinical benefit or no benefit) as a rationale for low-value care. Class II recommendations consider "net" clinical benefits by comparing risk or harms with benefits. Online Supplement Exhibit 1 provides a decision chart used in our assessment of the evidentiary rationale

followed by ACC/AHA ( $N=250,21 \%), \operatorname{ASCO}(N=184,16 \%)$, USPSTF ( $N=101,8.7 \%)$, and ACP ( $N=50,4.3 \%)$ (Fig. 2$)$. Most recommendations focused on the circulatory system $(N=325,28 \%)$ or neoplasms $(N=323,28 \%)$, but recommendations were available across all 21 ICD-10-CM disease chapters (Online Supplement Figure 2). Pharmaceuticals were most frequently cited as low-value care $(N=337,29 \%)$, followed by screening $(N=266,23 \%)$ and medical procedures $(N=214$, 18\%) (Online Supplement Figure 3).

The services deemed "low value" differed substantially across organizations. The only two low-value services listed by more than two organizations, for example, were prostate cancer screening for men aged over 70 (USPSTF, CW, ACP) and cervical cancer screening for women who had a hysterectomy (USPSTF, CW, ACP). Five additional low-value services were co-listed by two organizations, including cancer screenings for individuals with less than 10 years of life expectancy, cardiovascular disease screening for asymptomatic and low-risk adults, and sentinel lymph node biopsy to evaluate early, thin melanoma (Online Supplement Table 2).
We found relatively little overlap in recommendations across organizations. In an additional analysis of the 101 USPSTF Grade D (recommendation against, $n=30$ ) and Grade I (insufficient evidence, $n=71$ ) recommendations, only 12 Grade D and 8 Grade I recommendations appeared on the Choosing Wisely list. Of the 8 Grade C (use professional judgment and patient preference) USPSTF recommendations, two were not recommended by ACP due to low economic value. ACP's recommendations include prostate cancer screening for average-risk individuals aged 50 to 69 years who have not had an informed discussion and do not express a clear preference; and colorectal cancer screenings for average-risk individuals younger than 50 years or older than 75 years or those with an estimated life expectancy of fewer than 10 years. ${ }^{25,26}$

\section{Analysis of Evidentiary Rationale}

"No net clinical benefit" (37\%, 428 of 1167) and "little or no clinical benefit" $(25 \%, 296$ of 1167$)$ were the most commonly

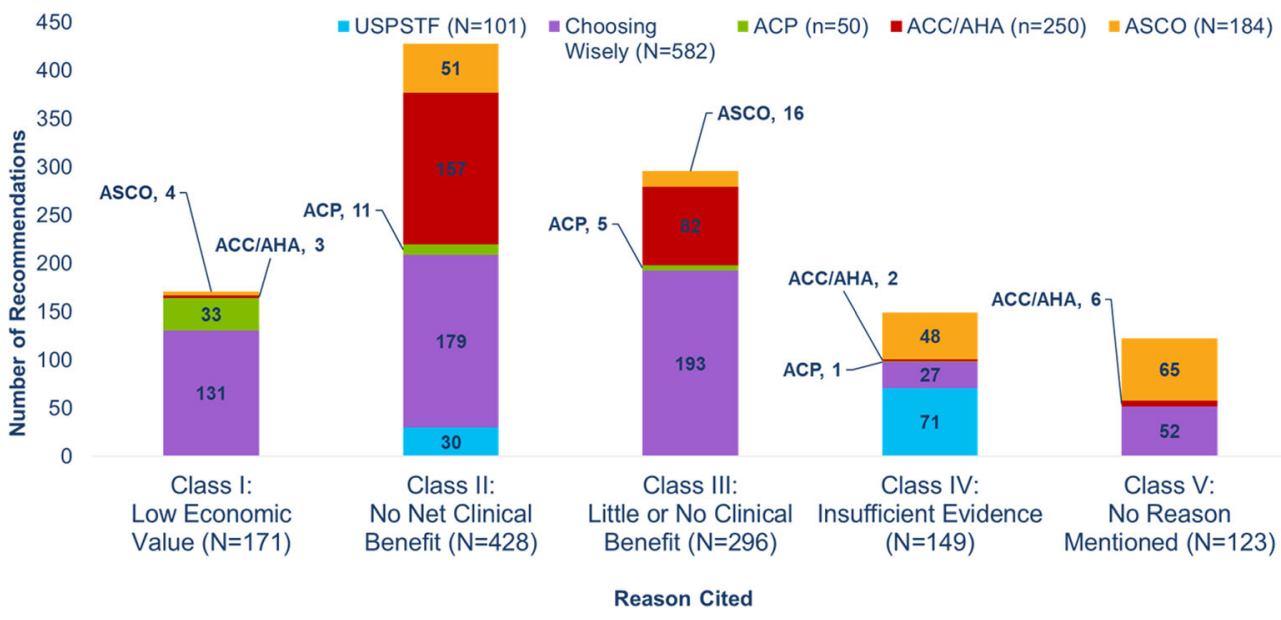

Figure 2 Evidentiary rationale of low-value care recommendations across five guidelines. Note: Authors' analysis of guideline recommendations from five organizations: US Preventive Services Task Force (USPSTF), Choosing Wisely (CW), American College of Physicians (ACP), American College of Cardiology/American Heart Association (ACC/AHA), and American Society of Clinical Oncology (ASCO). 
reported reasons for classifying an intervention as low value. A small percentage of guidelines $(11 \%, 123$ of 1167$)$ provided no reason for why an intervention was deemed low value (Fig. 2).

Consideration of economic value was less frequently reported. Excluding the USPSTF recommendations, which do not explicitly incorporate the financial costs of providing a service in its assessment of the balance of benefits and harms,${ }^{27}$ only 171 (16\%) of the 1066 remaining recommendations explicitly mentioned economic considerations. Although pharmaceuticals were the most common intervention type among all low-value care recommendations, economic evidence was seldom considered $(6.8 \%, 23$ of 337). In contrast, $30 \%$ (45 of 151) of recommendations against a diagnostic service cited economic evidence.

Also, substantial variation exists in reported evidentiary rationales across different guidelines (Fig. 3). Despite no mention of cost/value considerations in their methodological frameworks, ${ }^{28,29}$ Choosing Wisely $(n=131)$ and ACP $(n=33)$ account for $96 \%$ (164 of 171) of the recommendations in which economic factors were explicitly mentioned as part of their evidentiary rationales. In contrast, ASCO and ACC/AHA mentioned the consideration of economic factors in recommendations, ${ }^{30,31}$ but only $2.2 \%$ (4 of 184) and $1.2 \%$ (3 of 250 ) of their recommendations explicitly included economic factors. The USPSTF recommendations cited "No net clinical benefit" (30\%, 30 of 101) or "Insufficient Evidence" (70\%, 71 of 101), reflecting their policy of excluding explicit cost considerations. ${ }^{27}$

Among the seven low-value services listed by multiple organizations (Online Supplement Table 2), all of the ACP recommendations $(n=6)$ mentioned economic reasons as part of their evidentiary rationales. In contrast, the USPSFT recommendations $(n=3)$ always mentioned "no net clinical benefits" for the same low-value recommendations. We documented substantial variation in evidentiary rationales among different clinical societies making the same recommendations as the Choosing Wisely. For example, four clinical societies
(American Academy of Family Physicians, American College of Preventive Medicine, American Society of Clinical Oncology, and American Urological Association) listed prostate cancer screening for men aged over 70 as low-value care in the Choosing Wisely list. However, four different evidentiary rationales were stated, from "no reason mentioned" to "little or no clinical benefit" to "no net clinical benefit" to "low economic value."

Economic studies were sometimes cited in recommendations regardless of the stated evidentiary rationale. For example, 26\% (44 of 171) of the Class I recommendations (i.e., recommendations that explicitly mentioned economic reasons as part of its rationale) cited at least one economic study. In comparison, $21 \%$ (210 of 996) of the Class II-V recommendations (i.e., recommendations that did not explicitly state economic reasons) also cited such evidence. However, among the cited economic studies, the Class I recommendations were more likely to cite studies relevant to its specific recommendation (e.g., based on population and settings) than the Class II-V recommendations (98\% [43 of 44] versus 53\% [111 of 210]) (Fig. 4). We also found substantial differences in the economic evidence cited in low-value recommendations across the 5 organizations (Fig. 5).

\section{Analysis of Cost-effectiveness Evidence}

Among 133 recommendations with at least one available CEA relevant to the topic, $82 \%$ (109 of 133) were consistent with cost-effectiveness evidence (i.e., not cost-effective, dominated, or more cost-effective alternatives available) (Fig. 6). For recommendations with inconsistent cost-effectiveness evidence $(16 \%, 21$ of 133$)$, most of the cost-effectiveness evidence was outdated, or became available after publication of the recommendation; or studied in particular subgroups (e.g., when "women" is the target population specified in the recommendation, the CEA focused on women of a particular age group) (Online Supplement Table 3).

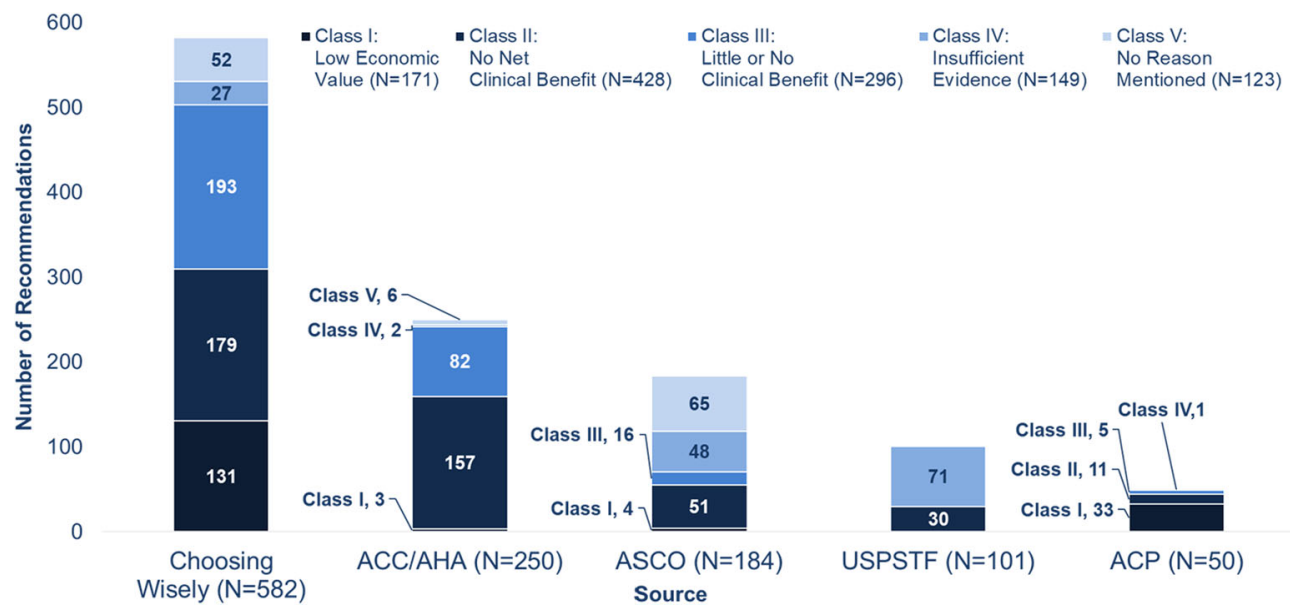

Figure 3 Evidentiary rationale reported in low-value recommendations by each organization. Note: The authors selected colors in this figure to be consistent with those of the classification of evidentiary rationale in Figure 1. 


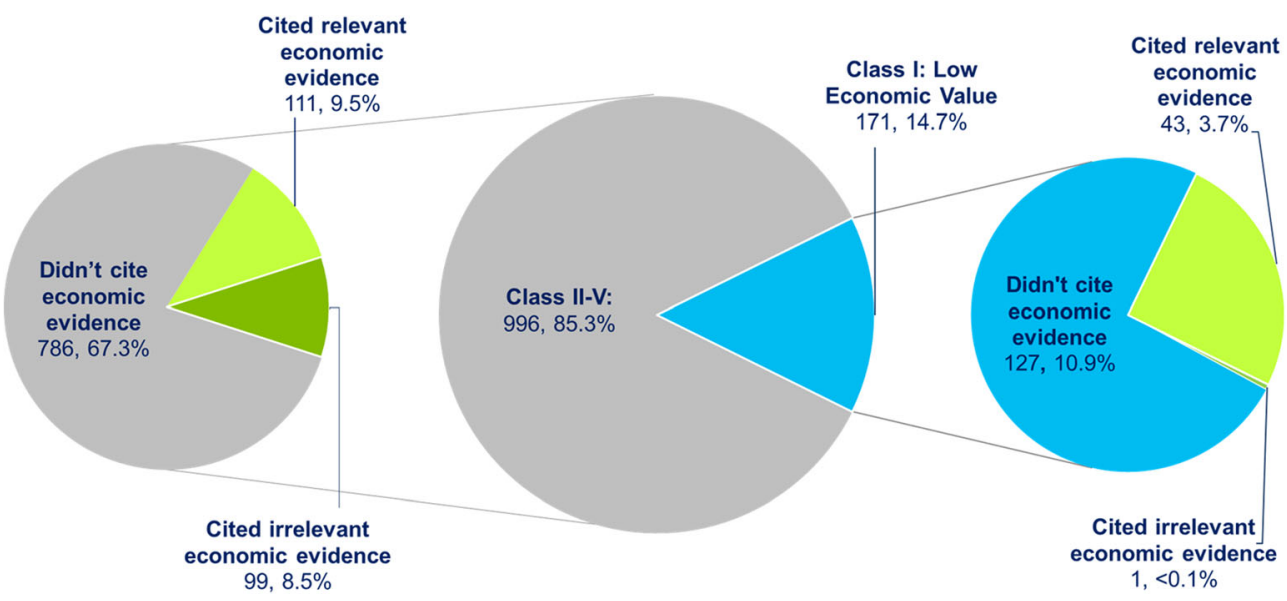

Figure 4 Inconsistency between the stated evidentiary rationale and the cited evidence in the low-value recommendations. Note: Class II through Class $\mathrm{V}$ represent recommendations that did not articulate economic concerns as a reason (e.g., unfavorable cost-effectiveness, high price, or related economic terms, including costs and efficiency) to support their low-value care recommendations. Although most of these recommendations did not cite any economic studies, a handful cited at least one study within their list of references. On the other hand, although Class I recommendations did articulate economic concerns as a reason for their recommendation, a handful of them did not cite any economic evidence on their list of references to support their claims.

\section{DISCUSSION}

After reviewing and classifying 1167 "low-value care" guideline recommendations, we found that the services deemed "low value" differed across organizations. Evidentiary rationales to support recommendations against an intervention's routine use also varied substantially across five US organizations. Moreover, the rationale for calling a service "low value" usually relied solely on clinical evidence rather than being inclusive of economic data. Only $16 \%$ of the recommendations explicitly mentioned economic factors as part of their rationale. However, when relevant cost-effectiveness studies were available, cost-effectiveness evidence was mostly consistent with guideline recommendations.

Inconsistency in guideline recommendations and evidentiary rationales is not just a pertinent issue for low-value care. Clinical guidelines in other areas, including those for hypertension, ${ }^{32}$ bipolar disorder, ${ }^{33}$ gout, ${ }^{34}$ and latent tuberculosis, ${ }^{35}$ are also inconsistent in their recommendations. The inconsistency reflects different processes, timing, and criteria used to develop guideline recommendations by organizations. For example, each specialty society's Choosing Wisely list differs in the process (e.g., the type and experience of experts, the use of external review, the involvement of patient representatives), the timing (e.g., dates of publication, frequency of update), and the criteria (e.g., clinical evidence of efficacy and/or harm, cost, the potential for overuse, prevalence of utilization), and the process. ${ }^{36}$ The inconsistency across guidelines could lead to variations in clinical practice, confusion among patients and clinicians, and the added challenges to de-implementing lowvalue care. ${ }^{37}$

Using the classification of the evidentiary rationale for lowvalue care (Fig. 1) along with the GRADE criteria for the quality of evidence, ${ }^{38}$ the development of a consensus grading structure on the strength and evidentiary rationale would help

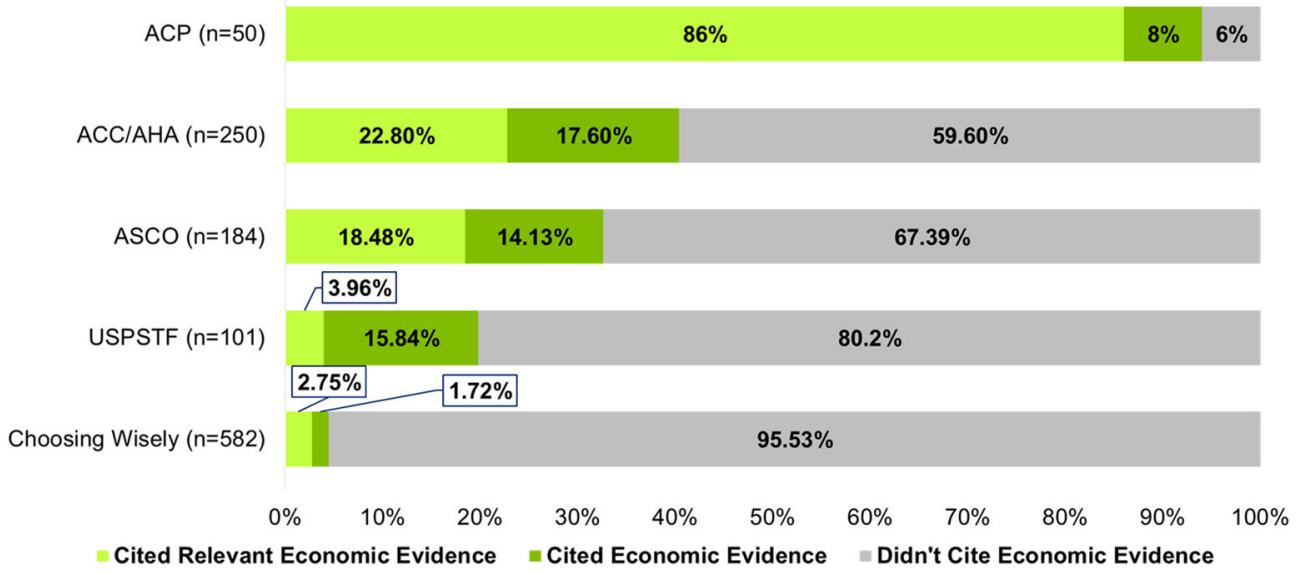

Figure 5 Economic evidence cited in low-value recommendations by each organization. Note: The type of economic evidence included empirical studies, systematic reviews, and meta-analyses of cost studies, cost-effectiveness analyses, and budget impact analyses. Among identified economic evidence, we determined whether the cited evidence was relevant to the specific recommendation based on the intervention and comparator(s), disease classification, population, and settings (e.g., hospital, nursing home). 

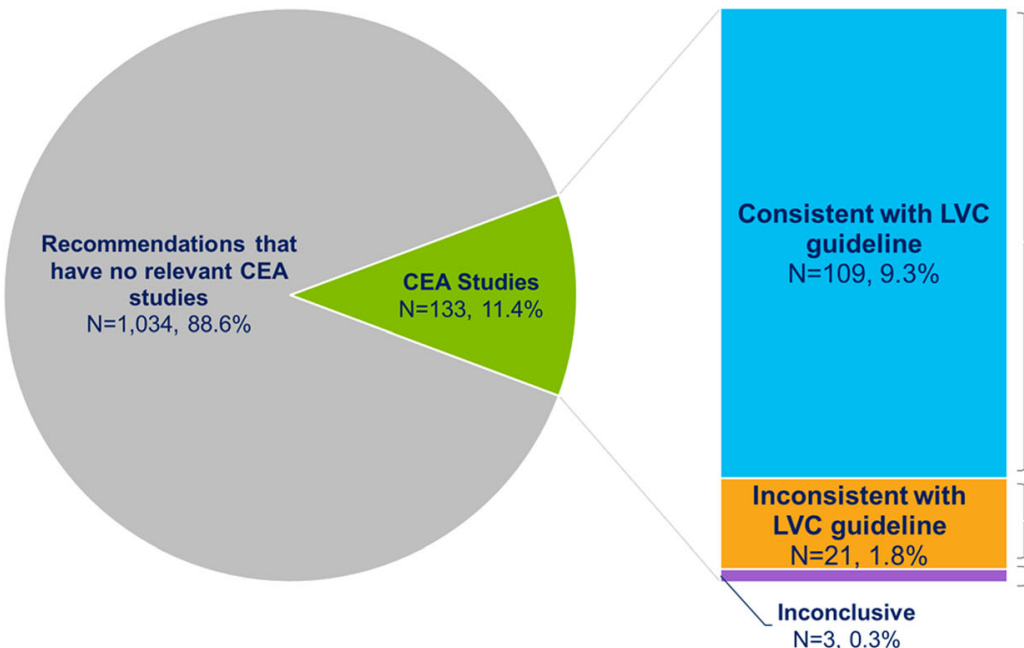

- Not cost-effective

- Dominated

- Comparator

- Cost-effective

- Cost-saving

- Multiple studies with conflicting results

Figure 6 Consistency between available cost-effectiveness evidence and low-value recommendations. Note: Authors' analysis of costeffectiveness studies cited in guideline recommendations from five organizations, supplemented by additional cost-effectiveness evidence available from Tufts Medical Center CEA Registry (www.cearegistry.org). We evaluated whether the cost-effectiveness evidence was consistent with the "low-value" care recommendation based on the following criteria: (1) when the intervention was less effective and more costly than its comparator (i.e., "dominated"); (2) when the intervention was deemed "not cost-effective"; or (3) when more cost-effective alternatives were available.

improve de-implementation efforts of low-value care. Moreover, an explicit and consistently applied grading and classification system across organizations could provide patients, clinicians, and others a greater understanding of the evidentiary rationale underlying the low-value care recommendation.

The lack of economic evidence considered in these guidelines could reflect that clinical guidelines seek to provide clinically relevant information, instead of costs- and reimbursement-related information. ${ }^{39}$ Also, the lack of cited economic evidence among Choosing Wisely recommendations might reflect the limited number of relevant references allowed. Some clinical societies often list a limited set of 5 or 10 references, and economic evidence might be less prioritized. Finally, despite its explicit statement of no economic evidence considered, the USPSTF recommendations often cite cost-effectiveness modeling studies. The USPSTF guideline committee might be considering non-economic evidence (e.g., modeled long-term impacts on health outcomes) presented in the cited studies.

While little consensus on the meaning of "value" exists in health care ${ }^{40,41}$ the medical professionals often define value as net clinical benefits, ${ }^{42,43}$ and if there is no net benefit to an intervention, the cost component is deemed less important. While examining clinical evidence remains essential, a sole focus on clinical evidence limits the classification of low-value care to clinically unnecessary and potentially harmful "novalue" services. Still, when there are widespread marginally effective clinical interventions that are simply too expensive for their relatively small clinical benefit, ignoring the cost component from the value consideration could be problematic.

A 2002 report on "Medical Professionalism in the New Millennium: A Physician Charter," one of the early antecedents of the Choosing Wisely initiative, emphasized the professional commitment to a just distribution of finite resources stating "physicians are required to provide health care that is based on the wise and cost-effective management of limited clinical resources". ${ }^{44}$ Broadening the evidence base to incorporate economic evidence, ${ }^{45}$ such as evidence from CEA, can help refine the definition of "low-value" care to reflect whether the costs of the intervention are worth the benefits.

Our study has limitations. We selected the five US-based guidelines in our sample to provide a thorough representation of low-value care recommendations across disease area, intervention type, and target population. However, this is not an exhaustive list, and our study is not a systematic review of all current low-value care guidelines. The addition of other clinical guidelines, including non-US-based guidelines, may provide a more comprehensive understanding of the consistency and the underlying evidentiary rationale across various lowvalue care guidelines.

Our assessments were solely based on information reported in the most recently published guidelines, including recommendation statements and cited references, as opposed to an independent review of the evidence base. Guideline committees might have considered additional evidence that was not cited in their recommendation statement. Also, subsequently published studies would not be cited in the original recommendation guidelines. It is likely that more economic evidence is available for some of the low-value recommendations, but our study aimed to investigate the evidentiary rationale to support the recommendations at the time of the publication.

Finally, we relied on the authors' reported costeffectiveness judgments to determine consistency with lowvalue care recommendations because of the different timing and settings of the original study. When we re-evaluated the cost-effectiveness evidence with a common \$100,000/QALY threshold in the US setting, ${ }^{46-50}$ eight low-value recommendations changed from consistent to inconsistent (i.e., deemed 
cost-effective under the $\$ 100,000 /$ QALY threshold, instead of the author's judgment) while one recommendation changed from consistent to inconsistent (i.e., being cost-effective). The overall result is slightly changed from 109 to 102 low-value care recommendations that were consistent with costeffectiveness evidence.

\section{CONCLUSION}

Our analysis indicates that low-value care guidelines are often inconsistent and mostly rely on clinical rather than economic evidence. While examining clinical evidence remains essential, broadening the evidence base to incorporate cost-effectiveness evidence can help refine the definition of "low-value" care to reflect whether an intervention's costs are worth its benefits. Developing a consensus grading structure on the strength and evidentiary rationale of low-value recommendations would be a step forward for improving de-implementation efforts of lowvalue care.

Corresponding Author: David D. Kim, PhD; Center for the Evaluation of Value and Risk in Health, Institute for Clinical Research and Health Policy Studies, Tufts Medical Center, 800 Washington St., Box 063, Boston, MA 02111, USA (e-mail: dkim3@tuftsmedicalcenter.org).

Funding This project was supported by a research grant from Arnold Ventures (formerly, the Laura and John Arnold Foundation).

\section{Compliance with ethical standards:}

Conflict of Interest: All of the authors are supported by a research grant from Arnold Ventures. DDK, LAD, ATD, JDS, DAO, and PJN are employees of the Center for the Evaluation of Value and Risk in Health (CEVR) at Tufts Medical Center, which maintains the CEA Registry used as a data source. The CEA Registry is supported by subscription revenue from academic institutions, government agencies, and pharmaceutical and device companies. Dr. Wong is a member of the US Preventive Services Task Force.

Disclaimer: This article does not necessarily represent the views and policies of the USPSTF. The funder had no role in study design, data collection and analysis, the decision to publish, and preparation of the manuscript.

\section{REFERENCES}

1. Shrank WH, Rogstad TL, Parekh N. Waste in the US Health Care System: Estimated Costs and Potential for Savings. JAMA. 2019.

2. Center for Value-Based Insurance Design 2020;Pageshttps://vbidcenter. org/initiatives/low-value-care/ on June 292020.

3. Brownlee S, Chalkidou K, Doust J, Elshaug AG, Glasziou P, Heath I, et al. Evidence for overuse of medical services around the world. Lancet. 2017;390(10090):156-68.

4. American Board Of Internal Medicine (ABIM) Foundation;Pageshttps:// www.choosingwisely.org/ on November 272020.

5. Elshaug AG, McWilliams JM, Landon BE. The value of low-value lists. JAMA. 2013;309(8):775-6.

6. Rosenberg A, Agiro A, Gottlieb M, Barron J, Brady P, Liu Y, et al. Early Trends Among Seven Recommendations From the Choosing Wisely Campaign. JAMA Intern Med. 2015;175(12):1913-20.
7. Carter EA, Morin PE, Lind KD. Costs and Trends in Utilization of Lowvalue Services Among Older Adults With Commercial Insurance or Medicare Advantage. Med Care. 2017;55(11):931-9.

8. Chambers JD, Salem MN, D'Cruz BN, Subedi P, Kamal-Bahl SJ, Neumann PJ. A Review of Empirical Analyses of Disinvestment Initiatives. Value Health. 2017;20(7):909-18.

9. Schwartz AL, Landon BE, Elshaug AG, Chernew ME, McWilliams JM. Measuring low-value care in Medicare. JAMA Intern Med. 2014;174(7):1067-76.

10. Schwartz AL, Zaslavsky AM, Landon BE, Chernew ME, McWilliams JM. Low-Value Service Use in Provider Organizations. Health Serv Res. 2018;53(1):87-119.

11. Colla CH, Morden NE, Sequist TD, Mainor AJ, Li Z, Rosenthal MB. Payer Type and Low-Value Care: Comparing Choosing Wisely Services across Commercial and Medicare Populations. Health Serv Res. 2018;53(2):73046.

12. Chua KP, Schwartz AL, Volerman A, Conti RM, Huang ES. Use of LowValue Pediatric Services Among the Commercially Insured. Pediatrics. 2016;138(6).

13. Schwartz AL, Jena AB, Zaslavsky AM, McWilliams JM. Analysis of Physician Variation in Provision of Low-Value Services. JAMA Intern Med. 2019;179(1):16-25.

14. Colla CH. Swimming against the current-what might work to reduce lowvalue care? N Engl J Med. 2014;371(14):1280-3.

15. Powers BW, Jain SH, Shrank WH. De-adopting Low-Value Care: Evidence, Eminence, and Economics. JAMA. 2020.

16. Kim DD, Ollendorf DA, Neumann PJ, Fendrick AM. Crisis into opportunity: can COVID-19 help set a path to improved health care efficiency? Am J Manag Care. 2020;26(9):369-70.

17. de Vries EF, Struijs JN, Heijink R, Hendrikx RJ, Baan CA. Are lowvalue care measures up to the task? A systematic review of the literature. BMC Health Serv Res. 2016;16(1):405.

18. Garner S, Docherty M, Somner J, Sharma T, Choudhury M, Clarke M, et al. Reducing ineffective practice: challenges in identifying low-value health care using Cochrane systematic reviews. J Health Serv Res Policy. 2013;18(1):6-12.

19. Gliwa C, Pearson SD. Evidentiary rationales for the Choosing Wisely Top 5 lists. JAMA. 2014;311(14):1443-4.

20. Viera AJ, Garrett JM. Understanding interobserver agreement: the kappa statistic. Fam Med. 2005;37(5):360-3.

21. Weinstein MC, Russell LB, Gold MR, Siegel JE. Cost-effectiveness in health and medicine: Oxford university press; 1996.

22. Neumann PJ, Sanders GD, Russell LB, Siegel JE, Ganiats TG. Costeffectiveness in health and medicine: Oxford University Press; 2016.

23. Neumann PJ, Sanders GD. Cost-Effectiveness Analysis $2.0 \mathrm{~N}$ Engl J Med. 2017;376(3):203-5.

24. Center for the Evaluation of Value and Risk in Health 2020;Pages. Accessed at Institute for Clinical Research and Health Policy Studies, Tufts Medical Center at www.cearegistry.org on May 202020.

25. Wilt TJ, Harris RP, Qaseem A. High Value Care Task Force of the American College of P. Screening for cancer: advice for high-value care from the American College of Physicians. Ann Intern Med. 2015;162(10):718-25.

26. Harris RP, Wilt TJ, Gaseem A. High Value Care Task Force of the American College of P. A value framework for cancer screening: advice for high-value care from the American College of Physicians. Ann Intern Med. 2015;162(10):712-7.

27. US Preventive Services Task Force 2015;Pageshttps://www. uspreventiveservicestaskforce.org/uspstf/sites/default/files/inlinefiles/procedure-manual-2020_3.pdf on July 102020.

28. Gaseem A, Kansagara D, Lin JS, Mustafa RA, Wilt TJ. Clinical Guidelines Committee of the American College of P. The Development of Clinical Guidelines and Guidance Statements by the Clinical Guidelines Committee of the American College of Physicians: Update of Methods. Ann Intern Med. 2019;170(12):863-70

29. Choosing Wisely 2020;Pageshttps://www.choosingwisely.org/our-mission/facts-and-figures/ on July 102020.

30. ACCF/AHA Task Force on Practice Guidelines, American College of Cardiology and American Heart Association, 2010;Pageshttps://www. acc.org/ /media/Non-Clinical/Files-PDFs-Excel-MS-Word-etc/Guidelines/About\%20Guidelines\%20and\%20Clinical\%20Documents/Methodology/2014/Methodology\%20Practice\%20Guidelines.pdf?la=en on July 102020 .

31. American Society of Clinical Oncology 2019;Pageshttps://www.asco.org/ sites/new-www.asco.org/files/content-files/practice-and-guidelines / documents/2019-Guidelines-Methodology-Manual.pdf on July 102020. 
32. Alper BS, Price A, van Zuuren EJ, Fedorowicz Z, Shaughnessy AF, Oettgen P, et al. Consistency of Recommendations for Evaluation and Management of Hypertension. JAMA Network Open. 2019;2(11):e1915975-e.

33. Parker GB, Graham RK, Tavella G. Is there consensus across international evidence-based guidelines for the management of bipolar disorder? Acta Psychiatrica Scandinavica. 2017;135(6):515-26.

34. Yu Y, Wang D, Zhou Q, Wang C, Ma X, Gao Y, et al. Recommendations in clinical practice guidelines on gout: systematic review and consistency analysis. Clinical and experimental rheumatology. 2020;38(5):964-72.

35. Hasan T, Au E, Chen S, Tong A, Wong G. Screening and prevention for latent tuberculosis in immunosuppressed patients at risk for tuberculosis: a systematic review of clinical practice guidelines. BMJ Open. 2018;8(9):e022445

36. Schpero WL. Limiting low-value care by "Choosing Wisely". Virtual Mentor. 2014;16(2):131-4.

37. Roman BR, Asch DA. Faded promises: the challenge of deadopting lowvalue care. American College of Physicians; 2014.

38. Balshem H, Helfand M, Schünemann HJ, Oxman AD, Kunz R, Brozek J, et al. GRADE guidelines: 3. Rating the quality of evidence. Journal of clinical epidemiology. 2011;64(4):401-6.

39. Burgers JS, Grol R, Klazinga NS, Mäkelä M, Zaat J, COLLABORATION FTA. Towards evidence-based clinical practice: an international survey of 18 clinical guideline programs. International Journal for Quality in Health Care. 2003; 15(1):31-045.

40. Porter ME. What is value in health care? N Engl J Med. 2010;363(26):2477-81.

41. Neumann PJ, Cohen JT. Measuring the Value of Prescription Drugs. N Engl J Med. 2015;373(27):2595-7.
42. Grady D, Redberg RF. Less is more: how less health care can result in better health. Archives of internal medicine. 2010;170(9):749-50.

43. Cassel CK, Guest JA. Choosing wisely: helping physicians and patients make smart decisions about their care. JAMA. 2012;307(17):1801-2.

44. American Board of Internal Medicine Foundation, American College of Physicians-American Society of Internal Medicine Foundation, European Federation of Internal Medicine. Medical professionalism in the new millennium: a physician charter. Ann Intern Med. 2002;136(3):243-6.

45. Pandya A. Adding Cost-effectiveness to Define Low-Value Care. JAMA. 2018;319(19):1977-8.

46. Neumann PJ, Cohen JT, Weinstein MC. Updating cost-effectiveness-the curious resilience of the \$50,000-per-QALY threshold. N Engl J Med. 2014;371(9):796-7.

47. Ryen L, Svensson M. The Willingness to Pay for a Quality Adjusted Life Year: A Review of the Empirical Literature. Health Econ. 2014.

48. Nimdet $\mathbf{K}$, Chaiyakunapruk $\mathbf{N}$, Vichansavakul $\mathbf{K}$, Ngorsuraches S. A systematic review of studies eliciting willingness-to-pay per qualityadjusted life year: does it justify CE threshold? PLoS One. 2015;10(4):e0122760.

49. Hirth RA, Chernew ME, Miller E, Fendrick AM, Weissert WG. Willingness to pay for a quality-adjusted life year: in search of a standard. Med Decis Making. 2000;20(3):332-42.

50. Vanness DJ. Lomas J, Ahn H. A Health Opportunity Cost Threshold for Cost-Effectiveness Analysis in the United States. Ann Intern Med. 2020.

Publisher's Note Springer Nature remains neutral with regard to jurisdictional claims in published maps and institutional affiliation. 